\title{
Influence of surface topography on brightness temperature of the regions along the road
}

\author{
Gang $\mathrm{Xu}^{1}$, Ying Yang ${ }^{*}$ and Zhiguo Meng ${ }^{2}$ \\ ${ }^{1}$ Tianjin Research Institute for Water Transport Engineering, M.O.T., Tianjin, 300456, China \\ ${ }^{2}$ College of Geoexploration Science and Technology, Jilin University, Changchun, Jilin, 130026, China
}

\begin{abstract}
Internal temperature of the road is one of the important indicators to evaluate the safety of the road, and the microwave radiometer data is only efficient way to acquire the internal temperatures. This study is to evaluate the influence of the surface topography on the brightness temperature (TB) measured the microwave radiometer data. The results are as follows. (1) The surface slope ( $\theta$ ) and its direction play the important roles on the TB. (2) The influence of $\theta$ on TB is weaker compared to that of the surface temperature. (3) At least in low latitude regions, the influence of topography on the TB can be neglected in macro scale. The conclusions are essential to better understand the internal physical parameters of the road with the microwave radiometer data.
\end{abstract}

\section{Introduction}

As a consequence of climate change, landslides, ice avalanches, and debris flows are becoming increasingly more problematic for the safety of the road [1-4]. Moreover, the deformation of the road surface is another important factor to damage the stability of the road [5]. Therefore, understanding the internal physical structures and the formation mechanisms has extremely important theoretical and practical value for ensuring the safe operation of the road [4-6].

The traditional methods to ensure the safe operation of the road are GPS and levelling surveys, which are also the most precise way to ensure the safe operation of the road. However, these methods have the following deficiencies. Firstly, a large number of human and material resources are dispensable to operate the works, and there still exist potential security risks. Secondly, the monitoring range is small, which is not suitable for rapid, accurate, and largescale monitoring. Thirdly, the observation cost is high, thus it is not suitable for long-term deformation monitoring of the roads with considerable distance.

As a new way, remote sensing technology has such advantages as wide monitoring range and high time resolution with a relatively low cost, which has been widely used to ensure the safe operation of the road. Over the past decades, a great deal of research has been carried out on the early identification of potentially dangerous sites, landslide monitoring, dynamic process modelling, and the rapid assessment of geohazards of the road [7-9]. In the regions along the roads, InSAR technology has been applied to reveal the initial deformation features of the road surface $[10,11]$, which can also provide the useful information to guide the rescue operations [4]. However, the InSAR technology is only sensitive to the surface that has been deformed, which yet has potential security risks for the operation of the roads.

The damage of the road begins with the change of the internal physical structures, which will directly alter the dielectric constant and temperature of the substrate. The passive microwave technology has been verified to be sensitive to the substrate dielectric constant and temperatures, which provides a potential way to evaluate the internal structures of the road. Moreover, the application of the passive microwave data is highly limited because of the poor knowledge regarding the triggering mechanism of the internal physical parameters, including the influence from the surface topography.

The fluctuation of the surface, represented by slope $(\theta)$ will directly alter the surface temperature distribution, which is widely accepted in studying the microwave thermal emission (MTE) of the terrestrial subjects $[12,13]$. Moreover, among the published researches relating to the brightness temperature, the surface roughness $(\sigma)$ also plays an important role to alter the effective emissivity, which will indirectly alter the MTE of the roads. However, the influence of the topography on the brightness temperature (TB) of the roads has not ever been evaluated. As the premise and foundation to evaluate the internal physical parameters with the microwave radiometer data, it is essential to study the influence of topography on the TB.

\section{Radiative transfer simulation}

The radiative transfer simulation is firstly employed to numerically evaluate the topography influence.

A usually used two-layered model is adopted to simulate the internal physical parameters of the road here. According to two-stream approximation principle, the TB,

*Corresponding author's e-mail: yangyinggis@foxmail.com 
emitted from the road with the incident angle $\theta 0$, consists of two parts [14]. One part is named TB1, radiation from the road material, and the other is named $\mathrm{TB} 2$, radiation from the road base. TB1 also comprises its upwelling radiation, T1up, and the downwelling, T1dn. That is,

$$
T_{B}=T_{B 1}+T_{B 2}=T_{1 u p}+T_{1 d n}+T_{2 u p} .
$$

Here

$$
d T_{1 u p}=\frac{1-r_{p 1}}{1-L} k_{a 1}(z) T(z) \sec \theta_{1} e^{-\int_{0}^{z} k_{a 1}\left(z^{\prime}\right) \sec \theta_{1} d z^{\prime}} d z
$$

$$
d T_{1 d n}=\frac{\left(1-r_{p 1}\right) r_{p 2}}{1-L} k_{a 1}(z) T(z) \sec \theta_{1} e^{-\left(\int_{z}^{d} k_{a 1}\left(z^{\prime}\right) \sec \theta_{1} d z^{\prime}+\int_{0}^{d} k_{a 1}\left(z^{\prime}\right) \sec \theta_{1} d z^{\prime}\right)} d z
$$

$d T_{2 u p}=\frac{\left(1-r_{p 1}\right)\left(1-r_{p 2}\right)}{1-L} k_{a 2}(z) T(z) \sec \theta_{2} e^{-\int_{d}^{z} k_{a 2}\left(z^{\prime}\right) \sec \theta_{2} d z^{\prime}} d z \cdot e^{-\int_{0}^{d} k_{a 1}\left(z^{\prime}\right) \sec \theta_{1} d z^{\prime}}$

$\theta_{1}$ and $\theta_{2}$ are the incident angles of the road and the road base, respectively. Guess the surface slope is $\theta$, the angles $\theta_{0}+\theta, \theta_{1}$ and $\theta_{2}$ follow the Snell law.

$T(z)$ is the temperature profile of the road material. To estimate $T(z)$, the effective solar illumination $S$ is strongly affected by the slope's component $\theta_{\phi}$ in longitude direction and $\theta_{\varphi}$ in latitude direction [15]. If $\phi$ ' and $\varphi^{\prime}$ are the corresponding solar angles of the measured unit along the longitude and latitude, respectively. Then, $S$ can be expressed as

$$
S=S_{0} \cos \left(\phi^{\prime}-\theta_{\phi}\right) \cos \left(\varphi^{\prime}-\theta_{\varphi}\right)
$$

$\mathrm{rp} 1$ is the effective reflectivity values of the free spaceregolith interface and $\mathrm{rp} 2$ is that of the regolith-rock interface, which are controlled by the dielectric constant of the lunar regolith and rock and strongly affected by the surface roughness $\sigma[14,16,17]$. For the dissipation of the road material, the variation of $\mathrm{rp} 2$ with interface roughness has slight effect on the final simulated TB [18] and the roughness effect on rp2 is neglected in this study. Only the influence of $\sigma$ on $\mathrm{rp} 1$ is considered. According to AIEM model and the random rough surface theory, rp1 which takes the surface roughness into attention is expressed as follows [16].

$$
r_{p 1}=r_{p} \cdot \exp [-(2 k s \cos \theta) 2]+\frac{1}{4 \pi \cos \theta} \int_{0}^{2 \pi} \int_{0}^{\pi / 2}\left[\sigma_{p p}\left(\theta, \theta_{j}, \phi_{j}\right)+\sigma_{p q}\left(\theta, \theta_{j}, \phi_{j}\right)\right] \sin \theta_{j} d \theta_{j} d \phi_{j}
$$

Where rp is the Fresnel reflection coefficients of the free space-regolith interface, $\mathrm{k}$ is the wavenumber in the free space, and $\mathrm{s}$ is the standard elevation deviation of the road surface which is directly related to the surface roughness $\sigma$.

$1 /(1-L)$ is multi-reflection coefficient.

Equation (1) to (r) are usual expression for the MTE of the road. The final $T_{B}$ is the integral of (2) over the whole thickness $d$ of the road material.

\section{Results}

To evaluate the influence of $\theta$ and $\sigma$ on MTE, the thickness layer of the road material is assumed to be $6 \mathrm{~m}$ with $390 \mathrm{~K}$ surface temperature. The temperature profile is calculated with thermal conductivity model, and the hyperbolic model is used as the density profile with depth. The dielectric constant of the road base is assumed $6.15+\mathrm{i} 0.155$. Then the variation of TB with $\theta$ and $\sigma$ in different conditions can be simulated with Eq.(1).

\section{$3.1 \theta$ Influence}

Figure. 1 shows the variation of the simulated TB with $\theta$. Figure. 1 indicates that TB changes apparently with $\theta$. With the increasing of $\theta$ from $0^{\circ}$ to $30^{\circ}$, the variations of TB is about $11 \mathrm{~K}$ at $3 \mathrm{GHz}$ and $15 \mathrm{~K}$ at $37 \mathrm{GHz}$, which is about $4 \%$ of the highest simulated $\mathrm{TB}$ of the correspondent frequency. This hints that the influence of $\theta$ on MTE is slightly. That is, $\theta$ may be one of the important influential factors for MTE, but not the decisive factor.

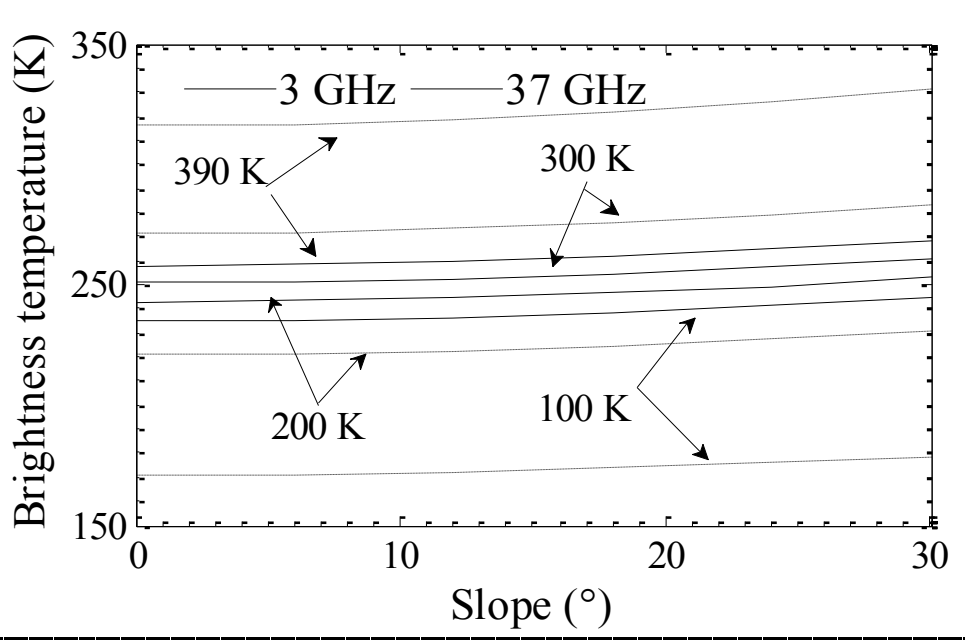

Figure 1. Variation of simulated brightness temperature with surface slope. 
Moreover, the surface temperature of the Moon varies greatly with the time and position (Heiken et al., 1991). Supposed that the surface temperature is $390 \mathrm{~K}, 300 \mathrm{~K}$ (daytime), $200 \mathrm{~K}$ (nighttime) and $100 \mathrm{~K}$ (nighttime), the variation of TB with $\theta$ is also simulated with Eq.(1) and the thermal conductivity model. Figure. 1 presents that the variation of TB with $\theta$ in the condition of $390 \mathrm{~K}$ surface temperature is up to $15 \mathrm{~K}$, which much more apparent than that in the $100 \mathrm{~K}$ surface temperature condition. This indicates that the influence of $\theta$ on MTE is strongly timedependent, and the surface illumination also plays an important role on this influence. Additionally, Figure.1 shows that the variation of TB with $\theta$ is much more apparent at high frequency at noon than that at low frequency. This hints that MTE at high frequency at noon will experience more strongly influence from $\theta$ than that at low frequency.

Furthermore, the change of TB resulted from the surface temperature is up to $152 \mathrm{~K}$, which is much higher than that brought by the variation of $\theta$. That is, the variation of the surface temperature will bring about more strongly changes of TB than that resulted from $\theta$, and $\theta$ must not be the decisive factor of the MTE of the lunar regolith.

\section{$3.2 \sigma$ Influence}

Surface roughness is another important factor that can affect MTE. The surface roughness is considered at a scale where the features involved are large compared to the used wavelength, and the advanced integral equation model (AIEM) is applicable in most conditions. Supposed that the surface temperature is $390 \mathrm{~K}, 300 \mathrm{~K}$ (daytime), $200 \mathrm{~K}$ (nighttime) and $100 \mathrm{~K}$ (nighttime), the variation of TB with $\sigma$ is simulated with Eq.(1) and the thermal conductivity model (Figure. 2).

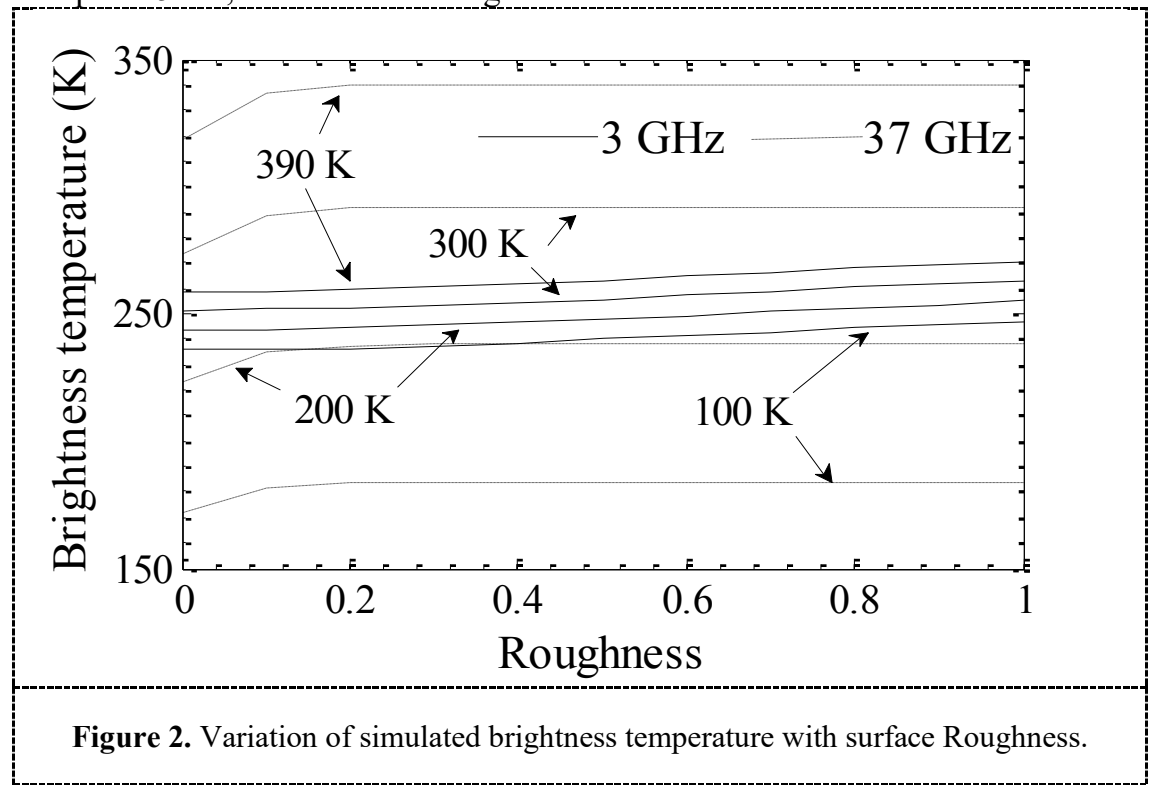

Figure. 2 indicates that the variation of TB with $\sigma$ is much more apparent at high frequency at noon than that at low frequency at midnight. This hints that the MTE at high frequency at noon will experience more strongly influence from $\sigma$ than that at low frequency at midnight. In high frequency, the variation of TB resulted from $\sigma$ is about 22 $\mathrm{K}$, while the change resulted from the surface temperature is up to $157 \mathrm{~K}$, which is much larger than that brought by $\sigma$. This again validates that the influence of the surface temperature on MTE is much stronger than that resulted from $\sigma$, and $\sigma$ is also not the decisive factor of MTE.

Moreover, Figure. 2 presents the variation of the simulated TB with $\sigma$, where $\theta$ is $0^{\circ}$. Figure 2 indicates that, with $\sigma$ increasing from 0 to 1 , the variation of TB is about $9 \mathrm{~K}$ at $3 \mathrm{GHz}$ and $12 \mathrm{~K}$ at $37 \mathrm{GHz}$, which is about $4 \%$ of the highest simulated TB of the correspondent frequency. This proportion is similar to that of $\theta$, which indicates that $\theta$ and $\sigma$ may play the similar role on MTE; while the small percentage hints that their influences on MTE are slight.

\section{Applications}

The study of the influence of the $\theta$ and $\sigma$ on $T_{B}$ is significant to better understand the internal physical parameters of the roads.

\subsection{Influence in Micro Scale}

To better understand the influence of the $\theta$ and $\sigma$ on $T_{B}$, the TB maps and the $\theta$ and $\sigma$ maps are generated in a pit region (Figure.3), which is widely distributed along the roads. Figure. 3 presents a distinctive perspective about the $T_{B}$ distribution. Here, the $T_{B}$ at the south wall of the pit is always high no matter at daytime or at nighttime, while the northern wall has the low $T_{B}$ throughout the day. The high $T_{B}$ is corresponding to the positive high $\theta$ at the south wall, while the low $T_{B}$ is corresponding to the negative high $\theta$ at the north crater wall. This also validates that $\theta$ and its direction play the important role on the $T_{B}$. 
However, there has not any relationship between $T_{B}$ and $\sigma$ in the pit region. That is, the micro scale $\sigma$ likely has no effect on the $T_{B}$ of the roads even in the pit regions.

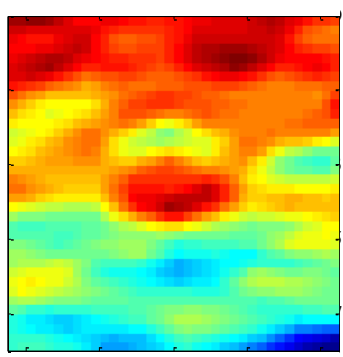

(a)

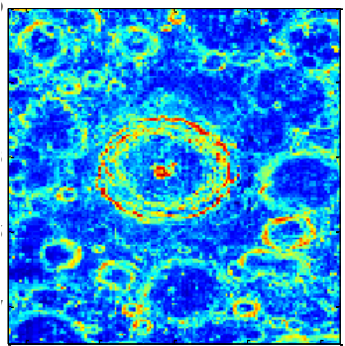

(c)

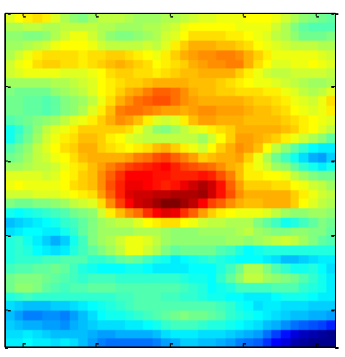

(b)

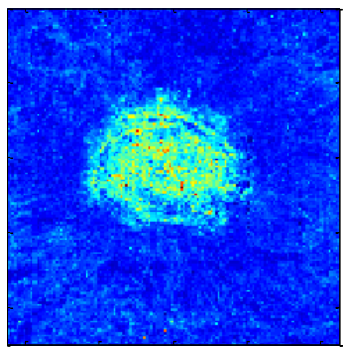

(d)

Figure 3. Distributions of TB, $\theta$ and $\sigma$ at a pit region: where (a) - (b) are the TB data at $3 \mathrm{GHz}$ (noon), $3 \mathrm{GHz}$ (midnight), respectively. (e) and (f) are the corresponding $\theta$ and $\sigma$ maps.

\subsection{Influence in Macro Scale}

The above discussion indicates that the $\theta$ is an essential parameter to impact the $T_{B}$ in the pit regions. However, in the region with great surface fluctuation (Figure. 4), there occurs a special phenomenon about our understanding of the $\theta$ influence.

Here, the diameter of the pit is more than $500 \mathrm{~km}$, and the change of the surface elevation is up to $10.4 \mathrm{~km}$, which provides a proper region to evaluate the influence of the $\theta$ on the $T_{B}$. The $T_{B}$ maps are at 12:00 (noon) and 1:00 (midnight), respectively (Figure. 4). Figure. 4 shows that the strong fluctuation of the ring structures and the relatively flat floor are clearly presented in the pit. However, the $T_{B}$ performance is practically unrelated to the topography here, probably suggesting that the $\theta$ has little or no influence on the TB in macro scale.

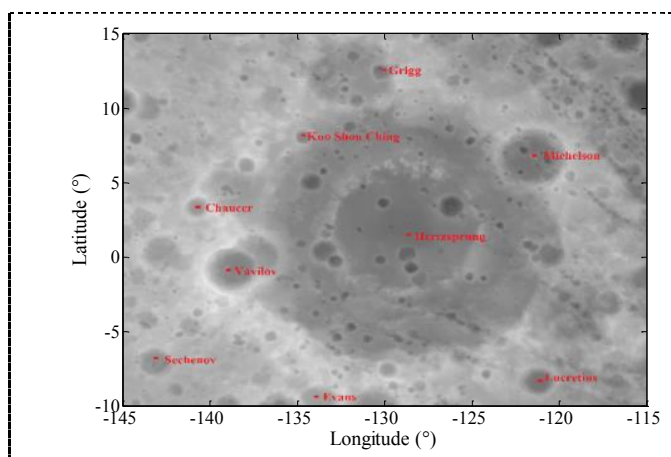

(a)

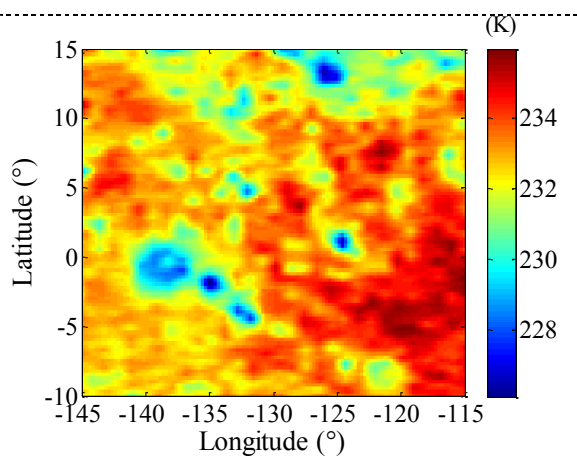

(b)

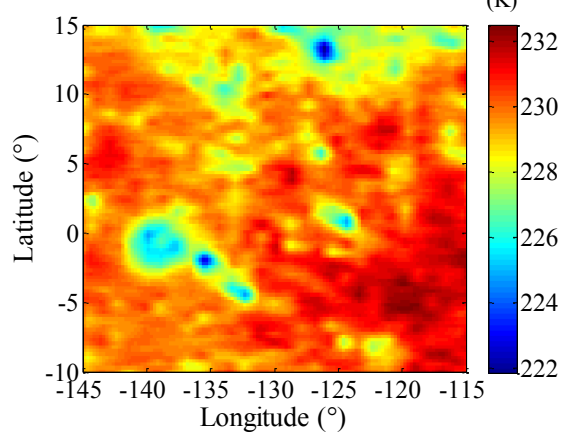

(c)

Figure 4. Geographic map (a),3.0 GHz (noon) (b) and 3.0 GHz (night) (c) maps in a large pit.

\section{Conclusions}

In this study, the influence of the surface topography on the TB is studied with. The global distributions of $T_{B}$ and $\theta$, the values along latitude $0^{\circ}$ and longitude $0^{\circ}$, and the statistical analysis all indicate that $\theta$ and its direction play the important role on the $T_{B}$. Secondly, the influence of $\theta$ on $T_{B}$ is weaker compared to that of the surface temperature.
Thirdly, after comparison the $T_{B}$ performances to the surface topography, the typical craters as Tycho and Aristarchus is not proper to study the rock influences, whose $T_{B}$ is controlled by the surface topography. Fourthly, at least in low latitude regions, the influence of topography on the $T_{B}$ can be neglected in macro scale.

The conclusions are essential to estimate the lunar regolith parameters with the CELMS data to, where $\theta$ and its direction must be paid fully attention. 
In this paper, to solve the problem that the application of the microwave radiometer data are strongly restricted by the poor knowledge about the surface topography in better understanding the internal physical parameters of the roads, the radiative transfer simulation and the AIEM model are firstly employed to study the variation of the TB with $\theta$ and $\sigma$ in the conditions of different frequency and surface temperature. The simulation results indicate that the influences are strong and they are increasing with the observation frequency. Then the influences are analyzed using the microwave radiometer data and $\theta$ and $\sigma$ derived with the elevation data. The analysis results show that $\theta$ and $\sigma$ are important influential factors on MTE along the same latitude, but not the decisive factors over the whole Moon surface. Moreover, the influence of $\theta$ on MTE is highly time-dependent, which plays a stronger role on MTE than that of $\sigma$ at noon along the same latitude. The effect of $\sigma$ on MTE is slightly affected by the observation time. Furthermore, the influence of $\theta$ and $\sigma$ on MTE is presented in the format of the changed temperature profile within the lunar regolith resulted from the altered effective illumination or the effective reflectivity.

The conclusions are essential to estimate the internal physical parameters of the roads with the microwave radiometer data, which likely provides a new way to ensure the safe operation of the road.

\section{Acknowledgments}

We download the LOLA data from http://pdsgeosciences.wustl.edu/missions/lro/lola.htm. This work was supported in part by the central level, scientific research institutes for basic R \& D special foundation (Grants Nos. TKS180409 and TKS180401),

\section{References}

1. Bardou, E., Delaloye, R. (2004) Effects of ground freezing and snow avalanche deposits on debris flows in alpine environments. Nat Hazards Earth Syst Sci., 4(4): 519-530.

2. Zhang, W. (2013) The general situation of Tibet natural disaster and the development process of its disaster aid. J. Catastrophology, 28(4): 176-181.

3. Delaney, K.B., Evans, S.G. (2015) The 2000 Yigong landslide (Tibetan Plateau), rockslidedammed lake and outburst flood: review, remote sensing analysis, and process modelling. Geomorphology, 246: 377-393.

4. Ouyang, C.J., An, H.C., Zhou, S., Wang, Z.W., Su, P.C., Wang, D.P., Cheng, D.X., She, J.X. (2019) Insights from the failure and dynamic characteristics of two sequential landslides at Baige village along the Jinsha River, China. Landslides, 16(7): 1397-1414.

5. Liu, W., He S., Ouyang C.J. (2016) Dynamic process simulation with a savage-hutter type

a) model for the intrusion of landslide into river. J. Mt. Sci., 13(7):1265-1274.
6. Feng Z, Yin YP, Li B, Zhang M (2012) Mechanism analysis of apparent dip landslide of Jiweishan in Wulong, Chongqing. Rock Soil Mech., 33(9):2704-2712.

7. Lin, C.H., Lin, M.L. (2015) Evolution of the large landslide induced by Typhoon Morakot: a case study in the Butangbunasi river, southern Taiwan using the discrete element method. Eng. Geol., 197:172-187.

8. Xie, M.W., Huang, J.X., Wang, L.W., Huang, J.H., Wang, Z.F. (2016) Early landslide detection based on D-InSAR technique at the Wudongde hydropower reservoir. Environ. Earth Sci., 75(8):1-13.

9. Ouyang, C.J., Zhao, W., Xu, Q., Peng, D.L., Li, W.L., Wang, D.P., Zhou, S., Hou, S.W. (2018) Failure mechanisms and characteristics of the 2016 catastrophic rockslide at Su village, Lishui, China. Landslides, 15(7):1391-1400.

10. Perski, Z., Hanssen, R., Wojcik, A., Wojciechowski, T. (2009) InSAR analyses of terrain deformation near the Wieliczka Salt Mine, Poland. Eng. Geol., 106(1):58-67.

11. Bayer, B., Simoni, A., Mulas, M., Corsini, A., Schmidt, D. (2018) Deformation responses of slow moving landslides to seasonal rainfall in the Northern Apennines, measured by InSAR. Geomorphology, 308:293-306.

12. Florinsky, I.V., Kulagina, T.B., Meshalkina, J.L. (1994) Influence of topography on landscape radiation temperature distribution. Int. J. Remote Sensing, 15(16):3147-3153.

13. Shi, J.C., Du, Y., Du, J.Y., Jiang, L.M., Chai, L.N., Mao. K.B., Xu, P., Ni, W.J., Xiong, C., Liu, Q., Liu, C.Z., Guo, P., Cui, Q., Li, Y.Q., Chen, J., Wang, A.Q., Luo, H.J., Wang, Y.H. (2012) Progresses on microwave remote sensing of land surface parameters. Sci. China Earth Sci., 7:1052-1078.

14. Ulaby, F.T., Moore, R.K., Fung, A.K. (1981) Microwave Remote Sensing: Active and Passive. Addison-Wesley Publishing Company, Reading Mass.

15. Liao, Y. (2011) Distributed model and numerical calculation of lunar surface physical temperature. Dissertation for the Master Degree. Huazhong university of science and technology.

16. Chen, K.S., Wu, T.D., Tsang, L. (2003) Emission of rough surfaces calculated by the integral equation method with comparison to threedimensional moment method simulations. IEEE Geosci. Remote Sensing, 41(1):90-101.

17. Shi, J.C, Du, Y., Du, J.Y., Jiang, L.M., Chai, L.N., Mao, K.B., Xu Peng, Ni, W.J., Xiong, C., Liu, Q., Liu, C.Z., Guo, P., Cui, Q., Li, Y.Q., Chen, J., Wang, A.Q., Luo, H.J., Wang, Y.H. (2012) Progresses on microwave remote sensing of land surface parameters. Sci. China Earth Sci., 55:10521078 . 
18. Meng, Z.G. (2008) Lunar regolith parameters retrieval using radiative transfer simulation and look-up table Dissertation for the Doctoral Degree. Jilin University. 